\title{
Study on the Standard Mechanism for the Use of Funds by Credit Village Borrowers
}

\author{
Neng Jiang Haoming Liu \\ School of Finance, Guizhou University of Finance and Economics, Guiyang Guizhou \\ 550025, China
}

\begin{abstract}
In order to test the credit village mode can effectively regulate the behavior of the borrower's moral hazard, in this paper, by establishing a dynamic game model, the traditional mode of borrower credit loans and credit village funds use behavior selection are analyzed. The results show that the credit village under the "supervision, report the behavior, to punish behavior" existence and behavior using the borrower credit village has significant positive correlation between confirmed the credit village unique mode of operation characteristics can effectively prevent moral hazard of the borrower.
\end{abstract}

\section{Keywords}

Credit village; Moral hazard; Dynamic game; Earnings

\section{信用村借款人资金使用行为规范机制研究}

\author{
江能 刘浩敏 \\ 贵州财经大学金融学院, 贵阳 550025, 中国
}

摘要: 为了检验信用村模式能够有效规范借款人的道德风险行为, 本文通过建立动态博弯模 型，对传统信用贷款模式与信用村模式下的借款人资金使用行为选择情况进行分析。研究结 果表明：信用村下的 “监督行为、上报行为、惩罚行为” 的存在与信用村借款人资金使用行 为之间具有显著的正相关关系，证实了信用村模式的独特运行特点能够有效防范借款人的道 德风险行为。

关键词 : 信用村 ; 道德风险 ; 动态博弯 ; 预期收益

\section{1. 引言}

贷款资金的违约运用是引起借款 人出现道德风险问题最主要的因素之 一。鉴于低端信贷市场中存在的信息 不对称现象，且借款人普遍缺乏贷款 抵押物的现实情况使得传统信用贷款
模式下的借款人投资行为规范机制失 效。信用村作为金融产品的新型创新 成果，其借款人之间存在的 “监督行 为、上报行为、惩罚行为” 是降低信 息不对称的重要因素。然而, 对于该 模式是否降低了借款人道德风险行为 的目的, 需要进行更为深入的分析。 


\section{2. 传统信贷模式下的借款人投资行为}

假设一：在本文所研究的贷款资 金运用阶段，为了便于分析，本文将 信贷资金运营阶段的市场参与主体视 为银行 $(B)$ 、借款者 $(\mathrm{L})$ 、其他参 与人 $(\mathrm{Li})$ 三方, 且信贷市场参与者 均满足理性人假设。其中，其他参与 人 Li 包括村级组织和除借款人之外的 其他村民成员。假设二：借款者（L) 的相关信息在其与银行 (B) 中是非对 称的，在其与其他参与人 ( $\mathrm{Li}$ ) 之间 近乎是对称的。假设三：银行 B 的战 略集为 $\mathrm{S}_{\mathrm{B}}=\{\mathrm{Y}, \mathrm{N}\}$, 其中 “Y” 表示贷 款, “N” 表示不贷款; 其他参与人 $\mathrm{Li}$ 的战略集为 $\mathrm{S}_{\mathrm{Li}}=\{\mathrm{S}, \mathrm{NS}\}$, 其中 “ $\mathrm{S}$ ” 表示其对借款人获得贷款资金后对资 金使用状况进行监督, “NS” 表示其 对借款人获得贷款资金后对资金使用 状况不进行监督; 借款人的战略集为 $\mathrm{S}_{\mathrm{L}}=\left\{\mathrm{H}_{\mathrm{p}}, \mathrm{L}_{\mathrm{p}}\right\}$, 其中 $\mathrm{H}_{\mathrm{p}}$ 表示借款人将借 款资金用于高风险用途， $L_{\mathrm{p}}$ 则表示借 款人将借款资金用于低风险用途。假 设四：假定借款者向银行借入一个单 位的货币资金，期限为一年，传统个 人信用贷款模式下的利息为 $r_{\mathrm{H}}$ 。假定 高风险项目成功的收益为 $R_{H}$, 失败的 收益为 0 , 成功概率为 $P_{H}$; 低风险项目 成功的收益为 $R_{L}$, 失败的收益为 0 , 成 功的概率为 $P_{L}$, 且 $P_{H} \in[0,1]$ 、 $P_{L} \in[0,1]$ 。根据 “投资风险与所得收 益是对称的”这一原理 : $R_{H}>R_{L}>0,0 \leq P_{H} \leq P_{L} \leq 1$ 。为 了分析的简便, 我们假定 $R_{H} \cdot P_{H}=R_{L} \cdot P_{L}=\bar{R}$ 。假设五: 本文 只考虑借款者之间相互监督的状况, 在这里认为银行对借款人的资金使用 采取不监督的策略。假设六：其他参 与人 Li 从银行取得个人信用贷款投资 于生产项目所产生的收益为 A, 且其收 益不受借款者的资金使用行为及还款 状况的影响。
在博弯的第一阶段：银行首先决 定贷款给借款人资金。在博亦的第二 阶段：其他参与人 Li 决定是否对借款 者进行监督。由于在传统个人信用贷 款模式下各个借款人都是相互独立的 个体, 故 Li 必然不会选择监督, 即 $\mathrm{P}(\mathrm{S})=0, \mathrm{P}(\mathrm{NS})=1$ 。则在博亦第二阶 段中, Li 选择不监督。在博弯的第三 阶段：借款人决定将所贷资金用于何 种类型的用途, 即借款人是选择将资 金用于高风险的非标定用途还是用于 低风险的标定用途。

借款人将借款资金用于高风险用 途的期望收益为:

$$
E_{L}^{H P}=P_{H} \cdot\left(R_{H}-r_{H}\right)
$$

借款人 $\mathrm{L}$ 将资金用于低风险用途 的期望收益为:

$$
E_{L}^{L P}=P_{L} \cdot\left(R_{L}-r_{H}\right)
$$

在传统个人信用贷款模式下, 若 只考虑本模型中所列的假设条件, 其 他借款者 $\mathrm{Li}$ 的收益为固定值 $\mathrm{A}$, 即 $\mathrm{ELi}=\mathrm{A}$ 。

$$
\begin{aligned}
(2-1) & \text { 式 }-(2-2) \text { 式得: } \\
& E_{L}^{H P}-E_{L}^{L P} \\
& =P_{H} \cdot\left(R_{H}-r_{H}\right)-P_{L} \cdot\left(R_{L}-r_{H}\right) \\
& =r_{H} \cdot\left(P_{L}-P_{H}\right)
\end{aligned}
$$

由于 $r_{H}>0$, 且 $P_{L}>P_{H}$, 则 $E_{L}^{H P}-E_{L}^{L P}>0, E_{L}^{H P}>E_{L}^{L P}$ 。由此可 知, 在传统个人信用贷款的条件下, 受高收益的驱使, 农户借款人在获得 银行的贷款资金之后，会以较大的可 能性将资金用于高风险用途，这就极 大地增加了借款人道德风险发生的可 能性。

当农户借款者 $\mathrm{L}$ 选择将资金用于 高风险用途时，银行发放贷款的预期 收益为:

$$
E_{B}=P_{H} \cdot r_{H}
$$

而对于银行来说, 其期望收益为: 


$$
E_{B}^{e}=P_{L} \cdot r_{H}
$$

不难发现: $E_{B}<E_{B}^{e}$ (因 $0<P_{H}<P_{L}<1$ ) 。即在传统个人信 用贷款模式下，银行的预期收益是小 于其期望收益的。在这种情况下，银 行的最佳策略是减少甚至是拒绝向农 村居民发放贷款。因此可以得到结 论：在借贷双方信息不对称的情况 下，传统信用贷款中的借款人在资金 使用上具有选择高风险的非标定用途 的内在激励; 而贷款人则会面临着较 高的道德风险, 最终会导致贷款人惜 贷，信贷市场失灵，难于达到规范借 款人资金使用行为的目的。

\section{3. 信用村模式下的借款人投资行为}

假设七: 相比于传统个人信用贷 款模式而言，信用村模式下的一个货 币单位资金一年期的贷款利息为 $\mathrm{r}_{\mathrm{L}}$, 且 $0<r_{L}<r_{H}<R_{L}<R_{H}$ 。假设八: 为了分析的简便, 在本部分的分析中 将全村村民简单看作是两个个体, 即 $\mathrm{L}$ 和 $\mathrm{Li}$ 。在这两个参与人中, 若有一方 违约, 则该信用村将面临被摘牌的危 险。为便于分析, 本章以 $\mathrm{L}$ 借款、 $\mathrm{Li}$ 监督为例进行分析。假设九: 若借款 者遵守合同约定使用资金并按期还本 付息, 则 $\mathrm{L}$ 将得到 $\mathrm{S}$ 的额外收益; 若 未遵守合同约定, 则会遭受 $S^{\prime}$ 的损 失。从长远角度来看, $\mathrm{S}$ 与 $\mathrm{S}^{\prime}$ 的值很 大, 在这里视为随着时间的推移会不 断趋近 $+\infty$, 其中 $\mathrm{S}^{\prime}>>\mathrm{S}>0$ 。 $\mathrm{T}$ 表 示 $\mathrm{L}$ 守约给其他参与者 $\mathrm{Li}$ 带来的潜在 好处, $\mathrm{T}^{\prime}$ 表示 L 违约给其他借款者 $\mathrm{Li}$ 带来的潜在损失。同样地, $\mathrm{T}^{\prime}>>\mathrm{T}$ $>0$ 。 $\mathrm{M}$ 为借款人将贷款资金用于非标 定用途为其带来的心理满足、缓解资 金需求等方面的收益。若其他参与人 Li 对借款人进行监督并将其不合规的
经营行为上报银行, 则借款人将面临 的银行惩罚为 $\mathrm{b}, \mathrm{Li}$ 将面临 $\mathrm{F}$ 单位的 额外收益。假设十：各借款人之间的 监督并不是以 1 的概率出现, 且 $\mathrm{Li}$ 将 监督结果上报银行也并不是以 1 的概 率出现。为了分析的简便, 本文假设 在信用村中其他参与人若选择将监督 结果上报即会选择对借款人采取惩罚 措施。假定 Li 对借款人进行监督的概 率为 $\mathrm{p}$, 则不进行监督的概率为 $(1-$ p）；同样地, Li 将其监督结果上报银 行的概率为 $q$, 则不上报其监督结果的 概率为 $(1-q)$ 。其中, $p \in[0,1]$ 、 $q \in[0,1]$ 。

在博弯的第一阶段：银行首先决 定贷款给借款人资金。在博亦的第二 阶段：其他参与人 Li 决定是否对借款 者 $\mathrm{L}$ 的资金使用用途、生产经营状况 等方面进行监督。若不监督, 则直接 进入借款人的投资决策阶段；若进行 监督, 则进入其他参与人 $\mathrm{Li}$ 自身决策 的第二个阶段。在博弯的第三阶段: 这阶段分为两个部分, 其一是 $\mathrm{Li}$ 不进 行监督情况下的借款人投资于何种类 型的项目的决策阶段; 其二是在 Li 进 行监督下, $\mathrm{Li}$ 是否将自己的监督结果 报告给银行 (即实施社会惩罚) 的决 策。在博竕的第四阶段：参与人 $\mathrm{Li}$ 采 取监督行为时, 在 Li 选择上报监督结 果与不上报监督结果（即进行社会惩 罚与不进行社会惩罚) 这两种情况 下，借款者将所借资金投资于何种类 型项目。

\section{1. 参与人 B 的收益分析}

银行作为整个博弯过程开始的第 一阶段, 其发放贷款获取的收入是固 定利率水平下的贷款利息收入, 故其 只关心借款人的投资项目收益能否如 期支付借款利息支出。下面来探讨一 下银行在借款人采取不同的投资行为 时的期望收益情况:

若借款人将借款资金用于高风险 用途，则银行的期望收益为: 
$E_{B}^{H P}$

$=p q \cdot r_{L} \cdot P_{H}+p(1-q) \cdot r_{L} \cdot P_{H}+(1-p) \cdot r_{L} \cdot P_{H}$ $=r_{L} \cdot P_{H}$

若借款人将借款资金用于低风险 用途，则银行的期望收益为: $E_{B}^{L P}$

$=p q \cdot r_{L} \cdot P_{L}+p(1-q) \cdot r_{L} \cdot P_{L}+(1-p) \cdot r_{L} \cdot P_{L}$ $=r_{L} \cdot P_{L}$

根据上面两式可知: $E_{B}^{H P}<E_{B}^{L P}$ （因 $P_{H}<P_{L}$ ），即借款者将所借资 金用于高风险用途时, 银行的期望收 益要小于借款人用于低风险项目时的 期望收益。

\section{2. 参与人 $\mathrm{Li}$ 的收益分析}

借款者若将贷款资金用于高风险 用途, 参与人 $\mathrm{Li}$ 的期望收益为:

$$
\begin{aligned}
& E_{L i}^{H P} \\
& =\left[p q \cdot P_{H} \cdot(T+F)+p(1-q) \cdot P_{H} \cdot T+(1-p) \cdot P_{H} \cdot T\right] \\
& +\left[\begin{array}{l}
p q \cdot\left(1-P_{H}\right) \cdot\left(-T^{\prime}+F\right)+p(1-q) \cdot\left(1-P_{H}\right) \cdot\left(-T^{\prime}\right) \\
+(1-p) \cdot\left(1-P_{H}\right) \cdot\left(-T^{\prime}\right)
\end{array}\right] \\
& =p q \cdot F+P_{H} \cdot T+\left(P_{H}-1\right) \cdot T^{\prime}
\end{aligned}
$$

借款者 L 若将贷款资金用于低风 险项目, 参与者 $\mathrm{Li}$ 的期望收益为:

$$
E_{L i}^{L P}
$$$$
=\left[p q \cdot P_{L} \cdot(T+F)+p(1-q) \cdot P_{L} \cdot T+(1-p) \cdot P_{L} \cdot T\right]
$$$$
+\left[\begin{array}{l}
p q \cdot\left(1-P_{L}\right) \cdot\left(-T^{\prime}+F\right)+p(1-q) \cdot\left(1-P_{L}\right) \cdot\left(-T^{\prime}\right) \\
+(1-p) \cdot\left(1-P_{L}\right) \cdot\left(-T^{\prime}\right)
\end{array}\right]
$$$$
=p q \cdot F+P_{L} \cdot T+\left(P_{L}-1\right) \cdot T^{\prime}
$$

$$
\text { (3-3) 式 - (3-4) 式, 可得: }
$$

$E_{L i}^{H P}-E_{L i}^{L P}$

$=\left[p q \cdot F+P_{H} \cdot T+\left(P_{H}-1\right) \cdot T^{\prime}\right]$

$-\left[p q \cdot F+P_{L} \cdot T+\left(P_{L}-1\right) \cdot T^{\prime}\right]$

$$
=\left(P_{H}-P_{L}\right) \cdot\left(T+T^{\prime}\right)
$$

根 据 假 设九可以推出 :
$\left(P_{H}-P_{L}\right) \cdot\left(T+T^{\prime}\right)<0 \quad$ 则 $E_{L i}^{H P}-E_{L i}^{L P}<0$, 即 $E_{L i}^{H P}<E_{L i}^{L P}$ 。这意 味着对于其他借款人 $\mathrm{Li}$ 而言, 借款者 将借款资金用于高风险用途给其带来 的期望收益小于 L 将借款资金用于低 风险用途的期望收益, 即: 在信用村 模式下, 参与人 $\mathrm{Li}$ 更希望借款人将其 所借资金用于较低风险的标定用途。

下面对借款人在将贷款资金用于 不同用途的情况下, 参与人 Li 对借款 人采取不同的监督、上报措施对于自 身期望收益变动的影响进行分析:

（1）在借款人将借款资金用于高 风险用途的情况下，来考虑 Li 的期望 收益情况对自身采取监督和上报行为 的概率变动的反应程度:

在 (3-3) 式中对 Li 的监督概率 $\mathrm{p}$ 求偏导数, 得:

$$
\frac{\partial E_{L i}^{H P}}{\partial p}=q \cdot F
$$

在 (3-6) 式中, 再对 $\mathrm{Li}$ 上报银行 的概率 $\mathrm{q}$ 求偏导数, 得:

$$
\frac{\partial^{2} E_{L i}^{H P}}{\partial p \partial q}=F
$$

由上述 (3-6) 式和 (3-7) 式可知: (3-6) 式中, $\frac{\partial E_{L i}^{H P}}{\partial p}>0$ 。这意味着在借 款人将借款资金用于高风险用途的情 况下, 随着 Li 对借款人的借款资金使 用状况进行监督的概率的提高, $\mathrm{Li}$ 的 期望收益会呈现增加的趋势。在 (3-7) 式中, $\frac{\partial^{2} E_{L i}^{H P}}{\partial p \partial \mathrm{q}}>0$, 这意味着在借款人 将借款资金用于高风险用途的情况 下，若 Li 对借款人的借款资金使用状 况进行监督并将其监督结果上报银行 的概率都提高的话, 则其期望收益会 得到提高。并且, 由于 $\mathrm{q} \in[0,1]$, 我 们可以发现: $\frac{\partial^{2} E_{L i}^{H P}}{\partial p \partial q}>\frac{\partial E_{L i}^{H P}}{\partial p}$, 也就 是说当借款人将资金用于高风险的非 
标定用途时, 参与人 Li 对借款人采取 既监督又上报其监督结果措施给自身 带来的期望收益水平的提高程度要大 于 $\mathrm{Li}$ 采取只监督但不上报监督结果措 施带来的期望收益水平的提高程度。 因此，参与人 $\mathrm{Li}$ 会更愿意对 $\mathrm{L}$ 的资金 使用行为进行监督并上报其监督结 果。

（2）在借款人将借款资金用于低 风险用途的情况下，来考虑 $\mathrm{Li}$ 的期望 收益情况对监督和上报的概率变动的 反应程度:

在 (3-4) 式中对 $\mathrm{Li}$ 的监督概率 $\mathrm{p}$ 求偏导数, 得:

$$
\frac{\partial E_{L i}^{L P}}{\partial p}=q \cdot F
$$

在 (3-8) 式中, 再对 $\mathrm{Li}$ 上报银行 的概率 $\mathrm{q}$ 求偏导数, 得:

$$
\frac{\partial^{2} E_{L i}^{L P}}{\partial p \partial q}=F
$$

根据 (3-8) 式和 (3- 9) 式可以推 出: $\frac{\partial E_{L i}^{L P}}{\partial p}>0, \frac{\partial^{2} E_{L i}^{L P}}{\partial p \partial q}>0$, 且 $\frac{\partial^{2} E_{L i}^{L P}}{\partial p \partial q}>\frac{\partial E_{L i}^{L P}}{\partial p}$ 。从而也可以得出结 论：当借款人将贷款资金用于低风险 用途时, Li 对 L 既监督又上报其监督 结果给自身带来的期望收益水平的提 高程度要大于 Li 只监督但不上报监督 结果带来的期望收益水平的提高程 度。考虑到理性人假设, Li 会更愿意 进行监督并上报其监督结果。

综合以上两种情况: 无论借款者 将贷款资金运用于何种类型的用途， $\mathrm{Li}$ 对 L 的资金使用情况进行监督并将 监督结果上报银行所得到的期望收益 都是高于不监督或者监督但不上报的 期望收益的。因此, 在信用村模式 下, 理性的参与人 $\mathrm{Li}$ 会加大对借款人 的资金使用行为的监督，并会将其监 督结果上报银行。从而减轻了借款人 在取得贷款资金后滥用资金、违规用
款现象的发生。

（3）参与人 L 的收益分析

借款者将贷款资金用于高风险用 途的期望收益:

$$
\begin{aligned}
& E_{L}^{H P^{\prime}}=\left[\begin{array}{l}
p q \cdot P_{H} \cdot\left(R_{H}-r_{L}-S^{\prime}-b\right)+p(1-q) \cdot P_{H} \cdot\left(R_{H}-r_{L}\right) \\
+(1-p) \cdot P_{H} \cdot\left(R_{H}-r_{L}\right)
\end{array}\right] \\
& +\left[\begin{array}{l}
p q \cdot\left(1-P_{H}\right) \cdot\left(M-S^{\prime}-b\right)+p(1-q) \cdot\left(1-P_{H}\right) \cdot\left(M-S^{\prime}-b\right) \\
+(1-p) \cdot\left(1-P_{H}\right) \cdot\left(M-S^{\prime}-b\right)
\end{array}\right] \\
& =P_{H} \cdot\left(R_{H}-r_{L}-p q \cdot b-p q \cdot S^{\prime}\right)+\left(1-P_{H}\right) \cdot\left(M-S^{\prime}-b\right)
\end{aligned}
$$

借款者 L 将贷款资金用于低风险 用途的期望收益为:

$$
\begin{aligned}
& E_{L}^{L P^{\prime}}=\left[\begin{array}{l}
p q \cdot P_{L} \cdot\left(R_{L}-r_{L}+S\right)+p(1-q) \cdot P_{L} \cdot\left(R_{L}-r_{L}+S\right) \\
+(1-p) \cdot P_{L} \cdot\left(R_{L}-r_{L}+S\right)
\end{array}\right] \\
& +\left[\begin{array}{l}
p q \cdot\left(1-P_{L}\right) \cdot\left(S-b-S^{\prime}\right)+p(1-q) \cdot\left(1-P_{L}\right) \cdot\left(S-b-S^{\prime}\right) \\
+(1-p) \cdot\left(1-P_{L}\right) \cdot\left(S-b-S^{\prime}\right)
\end{array}\right] \\
& =P_{L} \cdot\left(R_{L}-r_{L}+S\right)+\left(1-P_{L}\right) \cdot\left(S-b-S^{\prime}\right)
\end{aligned}
$$

$$
\text { (3-10) 式 - (2-1) 式, 得: }
$$

$$
\begin{aligned}
& E_{L}^{H P^{\prime}}-E_{L}^{H P} \\
& =\left[P_{H} \cdot\left(R_{H}-r_{L}-p q \cdot b-p q \cdot S^{\prime}\right)+\left(1-P_{H}\right) \cdot\left(M-S^{\prime}-b\right)\right] \\
& -\left[P_{H} \cdot\left(R_{H}-r_{H}\right)\right] \\
& =P_{H} \cdot\left(r_{H}-r_{L}-p q \cdot b-p q \cdot S^{\prime}\right)+\left(1-P_{H}\right) \cdot\left(M-S^{\prime}-b\right) \\
& <0
\end{aligned}
$$

$$
\text { (3-10) 式 - (3-13) 式, 得: }
$$

$$
\begin{aligned}
& E_{L}^{H P^{\prime}}-E_{L}^{L P^{\prime}} \\
& =\left[P_{H} \cdot\left(R_{H}-r_{L}-p q \cdot b-p q \cdot S^{\prime}\right)+\left(1-P_{H}\right) \cdot\left(M-S^{\prime}-b\right)\right] \\
& -\left[P_{L} \cdot\left(R_{L}-r_{L}+S\right)+\left(1-P_{L}\right) \cdot\left(S-b-S^{\prime}\right)\right] \\
& =\left(P_{H}-P_{L}\right) \cdot\left(S^{\prime}+b-r_{L}\right)-P_{H} \cdot\left(p q \cdot b+M+p q \cdot S^{\prime}\right)+(M-S) \\
& <0
\end{aligned}
$$

则可以推断出: $E_{L}^{H P^{\prime}}<E_{L}^{H P}$, $E_{L}^{H P^{\prime}}<E_{L}^{L P^{\prime}}$ 。即: 在信用村模式下, 由于信用村贷款模式的特征以及参与 者 Li 的监督及上报违约情况的存在, 借款者将贷款资金用于高风险用途的 期望收益要小于传统个人信用贷款模 式下的期望收益; 且用于高风险用途 的期望收益要小于用于低风险用途的 期望收益。这使得理性的借款者此时 在考虑多种因素的情况下, 为了保障 自身稳定收益，会更愿意将借款资金 
用于低风险用途，从而减轻了资金使 用风险, 提高了还款成功的可能性, 降低了道德风险发生的可能性。

考虑参与主体 Li 的监督概率和上 报概率变动情况下对借款者的期望收 益变动的影响, 作如下分析: 首先, (3-10) 式对 $\mathrm{p}$ 求一阶偏导, 得: $\frac{\partial E_{L}^{H P^{\prime}}}{\partial p}=-q \cdot b P_{H}-q \cdot S^{\prime} \cdot P_{H}=-q \cdot\left(b+S^{\prime}\right) \cdot P_{H}$

(3-14) 式再对 q 求一阶偏导, 得:

$$
\frac{\partial^{2} E_{L}^{H P^{\prime}}}{\partial p \partial q}=-\left(b+S^{\prime}\right) \cdot P_{H}
$$

由 (3-14) 式和 (3-15) 式的结果可 以推断出: $\frac{\partial E_{L}^{H P^{\prime}}}{\partial p}<0, \frac{\partial^{2} E_{L}^{H P^{\prime}}}{\partial p \partial q}<0$, 且 $\left|\frac{\partial E_{L}^{H P^{\prime}}}{\partial p}\right|<\left|\frac{\partial^{2} E_{L}^{H P^{\prime}}}{\partial p \partial q}\right|$ 。这意味着: 在信 用村贷款模式下，当借款人将贷款资 金用于高风险用途时，参与主体 Li 对 借款人既监督又上报其监督结果给借 款人带来的期望收益水平的降低程度 要大于 Li 只监督但不上报监督结果给 自身带来的期望收益水平的降低程 度。

\section{4. 研究结论}

第一，在传统个人信用贷款模式 下，基于获得高期望收益的驱使，获 得借款资金的村民会将所借款项以较 高的概率用于高风险用途，这就给银 行放贷带来了很大的风险, 最终使得 银行采取信贷配给策略，使得农村金 融市场资金严重短缺、极大地制约了 农村经济的发展。第二, 在信用村模 式下，信息传递机制更为完善、社会 惩罚机制更具威慑力。参与人 Li 会在 银行放贷后对借款人的相关情况进行 监督, 并在必要时采取社会惩罚措施 和向银行上报其监督结果, 故银行在
放贷之后可从参与人 $\mathrm{Li}$ 那里得到有关 借款人的相关信息。此时，违约借款 人将面临来自银行的银行惩罚和来自 参与主体 Li 的社会惩罚。第三, 在信 用村模式下, 由于其完善的信息传递 机制与惩罚机制的存在，借款人在获 得银行的贷款资金后，会以更大概率 将资金用于低风险用途，使得有效防 范借款人道德风险成为可能, 达到了 该模式成立的目的。

\section{参考文献}

[1] 江能. 联保贷款运行机制研究 [D]. 昆明理工大学, 2008.

[2]江能, 刍平. 联保贷款借款人投资 行为规范机制研究 $[\mathrm{J}]$. 统计与决 策，2010，（8）.

[3] 盛光华, 庞英, 张志远. 农户小额 信用贷款道德风险的随机监管博弯 分析 $[J]$. 农村金融观察，2014，

(6).

[4]王曙光, 乔郁等. 农村金融学 [M]. 北 京大学出版社, 2008.

[5]叶银龙. 农村信用体系建设、信用 成果运用与信贷模式创新——以浙 江丽水农村金融改革试点为例 $[\mathrm{J}]$. 西南金融，2016，（3）.

[6]杨宗政. 甘肃定西 “西关信用村” 创建的实践及启示 $[\mathrm{J}]$. 西部金融, 2010，（3）.

[7] Aniket,K. Sequential Group Lending with Moral Hazard[J].LSE Working Paper,2004,10 (2):244-255.

[8] Hermes and Lensink. Peer Monitoring, Social Ties and Moral Hazard in Group Lending Programs: Evidence from Eritrea. World Development, Vol.33,No.1,2005.

[9] Joseph P. Kaboski and Robert M. Townsend The Impacts of Credit on Village Economies,April 9,2009. 